\title{
Fibrous Dysplasia of the Middle Turbinate: About A Case Report
}

\author{
H. EL HAMMAOUI", M. EL GHAIDI, H EL MORTAJI, D BASRAOUI, H. Jalal
}

Radiology Department, Mother and Child Hospital, CHU Med VI, Marrakech

DOI: $10.36347 /$ sjmcr.2020.v08i02.024

| Received: 01.01.2020 | Accepted: 08.01.2020 | Published: 21.02.2020

*Corresponding author: H. EL HAMMAOUI

\section{Abstract}

Fibrous dysplasia is a common benign fibro-osseous disease involving the flat bones, often affecting the bony structures of the skull and facial skeleton. Primary occurrence or secondary involvement of the nasal turbinate is not a common manifestation of the disease. Involvement of the inferior turbinate generally does not have specific management-related issues; however, involvement of the middle turbinate, especially the lateral lamella, can predispose to surgical morbidity during endoscopic surgical management. Clinical presentation, management and features of the disease on CT imaging are presented.

Keywords: Fibrous dysplasia, CT, MRI.

Copyright @ 2020: This is an open-access article distributed under the terms of the Creative Commons Attribution license which permits unrestricted use, distribution, and reproduction in any medium for non-commercial use (NonCommercial, or CC-BY-NC) provided the original author and source are credited.

\section{INTRODUCTION}

Fibrous dysplasia (FD) is a condition characterized by progressive replacement of the normal bony structures with benign cellular fibrous connective tissue, leading to disorganized structure of the bone. 1 Depending on the extent of involvement of the skeletal components, the disease can be categorized as monostotic (limited to a single bone) or polyostotic[1, 3 ]. The most common sites of involvement are the membranous bones of the pelvic girdle, femur and tibia. Skull is also a common site of involvement. Involvement of the nasal turbinate is infrequent [3, 5]. Isolated involvement of the inferior turbinate, in view of its anatomical relation, does not predispose to significant management problems. However, involvement of the middle and superior turbinate, and lateral and basal lamella creates a unique situation wherein there is involvement of the skull base structures, leading to increased surgical morbidity. Involvement of the lateral lamella, by virtue of its attachment to the cribriform plate and proximity to the ethmoidal arteries, predisposes to risk of damage to the floor of the anterior fossa and vascular injury during surgical or endoscopic intervention. We present the case of fibrous dysplasia of the middle turbinate in a 11 year old child.

\section{CASE REPORT}

- A 11-year-old male was admitted in our radiology department with difficulty in breathing through the nose for2 years.

- Physical examination found a hard mass protruding through the right nostril.

- Laboratory parameters, including levels of alkaline phos-phatase, were normal.

- $\quad$ The CT showed ( figure 1 ):

- a voluminous ground-glass bony lesion with frosted glass on the right nasal cavity which it widens, with a wide base of implantation at the medial wall of the orbit which is thinned and an intensive contrast uptake .

- It is responsible for an ostiomatal filling with fluid retention of the right maxillary sinus without visualization of the midlle turbinate.

- This mass is well limited measuring $34 \times 52 \times 47 \mathrm{~mm}$.

- She pushes the nasal septum to the left with no extension beyond the skull-base or into the orbit.

- The patient underwent endoscopic surgery under general anaesthesia and complete excision of the mass was performed

- The mass was adherent to the medial wall of the orbit, the posterior aspect of the septum and the medial surface of the inferior turbinate.

- Histopathological examination confirmed the diagnosis (figure 2). 


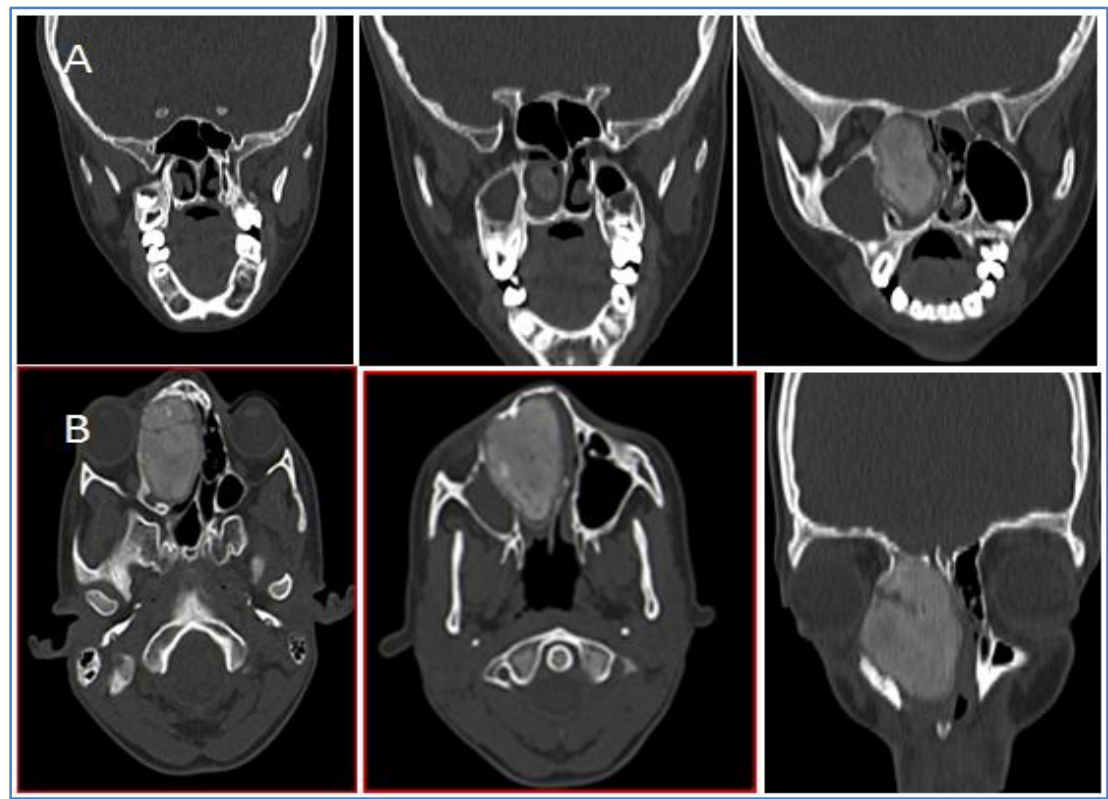

Fig-1: (a) Coronal b) Axial CT image at the mid-nasal cavity level demonstrates voluminous ground-glass bony lesion with frosted glass on the right nasal cavity which it widens, with a wide base of implantation at the medial wall of the orbit which is thinned

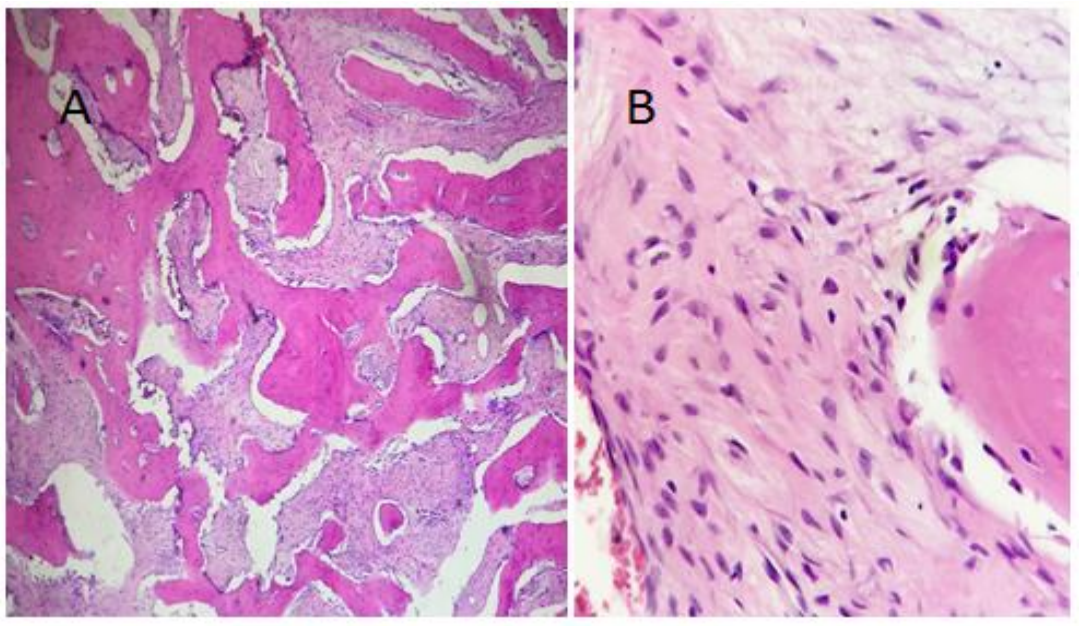

Fig-2: A: (HEX10): Primary bone streaks drawing broken rings and Chinese characters on a fibrous background. B: HEX40: bony spans without osteoblastic borders on a fibrous background sheltering a mononuclear inflammatory infiltrate and containing vessels Congestive blood

\section{DISCUSSION}

Localization of FD only at the middle turbinate is an extremely rare event; only two other cases having been reported in the English literature[2, 6]. Neither of which underwent surgical treatment. Nevertheless, the incidence of this disease is probably underestimated due to the asymptomatic bony proliferation that remains undetected until it gives rise to pathological fractures, pain due to neurological compression or functional disorders in the affected organ. Therefore, without related symptoms, diagnosis is usually incidental during radiological examinations performed for other causes.

On the middle turbinate, the bony degeneration gives rise to an increase in volume that can cause nasal ob- struction or alterations in sinus drainage. Videorhinoscopy shows turbinate enlargement that can be mistaken for a concha bullosa; this examination also allows evaluation of other, associated or correlated, sinus-nasal diseases. Axial-coronal CT, in thin sections, is the radiological exam of choice; it highlights the bony nature of the lesion with the charactestic "groundglass" appearance. The radiographic findings may change in relation to the evolutive stage of the disease, therefore, three classic radiological patterns have been described:

- Lytic or cysti-like form characterized by roundish rarefactive areas with a sclerotic border;

- Sclerotic form with homogeneous bone expansion;

- Pagetoid form with dishomogeneous areas of radiodensity and radiolucenty $[1,4]$.

- With CT, it is also possible:

- to define the boundaries and connections of the lesion;

- to exclude a polyostotic craniofacial form; 
- to identify other possible associated disorders;

- $\quad$ to monitor evolution of the disease.

Classically, on MRI, FD shows hypointensity in $T 1$ and $T 2$ weighted sequences, owing to a large fibrous component of the lesion. However, signal intensity of the bone lesions can vary depending on the composition of the bone and extent of mineralization.

FD has to be differentiated from other fibrodysplastic disorders (Paget disease, hyperparathyroidism, osteogenesis imperfecta ...) and from ossifying lesions (osteoma, osteosarcoma, chondroma, ossifying fibroma ...). To this end, histopathological studies are of fundamental importance since they reveal the presence of irregular bony trabeculae embedded in a cellular fibrous stroma without osteoblastic rimming 7. However, to distinguish diseases with such similar clinical and histological features, close cooperation between the clinician, the pathologist and the radiologist is essential 1, more especially for the ossifying fibroma that, unlike FD, usually occurs in adult age, is characterized by monostotic expansive lesions with a well-defined edge and, for this reason, shows a better response to surgical treatment.

FD may be associated with hormonal changes as well as defects in the calcium-phosphorus metabolism; therefore, blood tests such as calcaemia, alkaline phosphatase and parathormone assessment [6, 8] are mandatory to exclude a syndromic form, in particular those of McCune-Albright. On the contrary, the asso- ciation of FD with polypoid sinusitis, allergic bronchial asthma and ASA intolerance (Widal triad), that we consider incidental, has not been described, so far, in the literature.

No medical treatment has been found to be effective in the management of FD; therefore, in those cases presenting localized lesions with functional disorders or cosmetic alterations, surgical treatment is indicated. On account of the poorly defined limits between normal and dysplastic bone, "en-bloc" excision is often very difficult and associated with recurrence in approximately $20-25 \%$ of these patients. Partial resections or bony reshapes are more frequently performed also in consideration of the fact that the disease tends to exhaust itself after puberty.

Surgical treatment, particularly if not radical, re- quires long-term follow-up in order to monitor the risk of recurrence and to evaluate possible malignant degeneration. Indeed, FD is associated with sarcomatous evolution in $0.5-1 \%$ of cases, in particular in cases of craniofacial lesions [9, 10]. The risk of mali nant degeneration increases considerably after radiationtherapy that must be avoided in all cases.

In conclusion, Even though exceptional, localization of FD at the middle turbinate has been described, and, therefore, must be borne in mind in the differential diagnosis of the craniofacial ossifying disorder 24 . Likewise, pos- sible syndromic associations should be sought.

Videorhinoscopy and craniofacial CT are fundamen- tal examinations in the correct assessment of this dis- ease, diagnosis of which, however, depends on a scrupulous study of the histopathological features.

\section{REFERENCES}

1. Giannì AB. Malattie non neoplastiche non infiammatorie. In: Brusati R, Chiapasco M, editors. Elementi di Chirurgia Oro-Maxillo-Facciale. Milan: Ed. Masson. 1999: 141-52.

2. Alba JG, Armengot MC, Pérez CF, Díaz AF, Taleb C, Campos AC, Basterra JA. An exceptional form of cranio-facial fibrous dysplasia: fibrous dysplasia of middle turbinate. Acta otorrinolaringologica espanola. 2002 Apr;53(4):291-4.

3. Choen MM Jr, Howell RE. Etiology of fibrous dysplasia and McCune-Albright syndrome. Int J Oral Maxillofac Surg. 1999;28:5366-71.

4. Ozbek C, Aygenc E, Tuna EU, Fidan F, Ozdem C. Fibrous dysplasia of the temporal bone. Annals of Otology, Rhinology \& Laryngology. 2003 Jul;112(7):654-6.

5. Furin MM, Eisele DW, Carson BS. McCuneAlbright syndrome (polyostotic fibrous dysplasia) with intracranial frontoethmoid mucocele. Otolaryngology--Head and Neck Surgery. 1997 Apr;116(4):559-62.

6. LaBagnara Jr J, Yuppa F, Klein MH. Fibrous dysplasia of the middle turbinate with primary hyperparathyroidism. Ear, nose \& throat journal. 1998 Jul 1;77(7):552.

7. Tsai TL, Ho CY, Guo YC, Chen W, Lin CZ. Fibrous dysplasia of the ethmoid sinus. Journalchinese medical association. 2003 Mar;66(2):1313.

8. Caudill R, Saltzman D, Gaum S, Granite E. Possible relationship of primary hyperparathyroidism and fibrous dysplasia: report of case. Journal of oral surgery (American Dental Association: 1965). 1977 Jun;35(6):483-90.

9. Schwarz DT. The malignant transformation of fibrous dysplasia. Am J Med Sci. 1964;247:35-54.

10. Shapeero LG, Vanel D, Ackerman LV, TerrierLacombe MJ, Housin D, Schwaab G, Sigal R, Masselot J. Aggressive fibrous dysplasia of the maxillary sinus. Skeletal radiology. 1993 Nov 1;22(8):563-8. 